\title{
Water in Protoplanetary Disks
}

\author{
H. Nomura ${ }^{1}$, C. Walsh ${ }^{2}$, D. Heinzeller ${ }^{3}$ and T. J. Millar ${ }^{2}$ \\ ${ }^{1}$ Dept. of Astronomy, Graduate School of Science, Kyoto University, Kyoto 606-8502, Japan \\ email: nomura@kusastro.kyoto-u.ac.jp \\ ${ }^{2}$ Astrophysics Research Centre, School of Mathematics and Physics, Queen's University \\ Belfast, University Road, Belfast, BT7 1NN, UK \\ ${ }^{3}$ MetService Ltd., 30 Sallamanca Rd. Kelburn, Wellington 6012, New Zealand
}

\begin{abstract}
Infrared water line emission from protoplanetary disks, recently observed by the Spitzer and Herschel space telescopes, is thought to trace the surface layer of the inner to outer regions of the disks. We have modelled the water abundance profile and line emission, especially focusing on the effects of dust size growth and turbulent mixing. Comparison between model calculations and observations suggests a small grain model with turbulent mixing is preferred.
\end{abstract}

Keywords. line: formation, molecular processes, planetary systems: protoplanetary disks

\section{Introduction}

Planets are believed to form in protoplanetary disks. The Earth's oceans are thought to have been delivered to Earth by the impact of small icy bodies, such as comets, which were formed in the outer proto-Solar nebula. In this work, we have studied general processes of water formation in protoplanetary disks. Water emission lines have been detected recently from disks at wavelengths of $10 \mu \mathrm{m}$ to $540 \mu \mathrm{m}$ by the Spitzer and Herschel space telescopes and they are thought to originate from the surface layer at a radius of between about $1 \mathrm{AU}$ to $100 \mathrm{AU}$ from the central star. Here, we model the water line emission from a protoplanetary disk and compare the results with observations to investigate the physical conditions as well as the process of water formation in the disk.

\section{Physical and chemical models of protoplanetary disks}

The water abundance in the disk is calculated by running a pseudo-time-dependent chemical model at each grid point in our disk model. The gas-phase chemical reactions are from the UMIST Database for Astrochemistry (Rate06) to which we have added grain-surface reactions. Detailed photochemistry and X-ray ionization rates are taken into account. For gas-grain interactions, gas accretion onto grains as well as thermal and non-thermal desorption of icy mantle species, such as cosmic-ray- and photo-desorption, are considered (see Walsh et al. 2010, 2012 for details). The physical structure of the disk is calculated assuming static conditions. The gas density profile is obtained by assuming hydrostatic equilibrium and the gas temperature is calculated by assuming local thermal balance, taking into account the UV and X-ray irradiation from the central star. Here, we adopt a typical $\mathrm{T}$ Tauri star as the central star.

\section{Water formation and abundance profile in the disk}

Gas-phase water in protoplanetary disks is thought to form mainly in the following two ways; in the hot inner disk $\left(T_{\text {gas }}>300 \mathrm{~K}\right)$ water is formed very efficiently through reactions which possess an energy barrier,

$$
\mathrm{H}_{2}+\mathrm{O} \rightarrow \mathrm{H}+\mathrm{OH}, \quad \mathrm{H}_{2}+\mathrm{OH} \rightarrow \mathrm{H}+\mathrm{H}_{2} \mathrm{O} .
$$



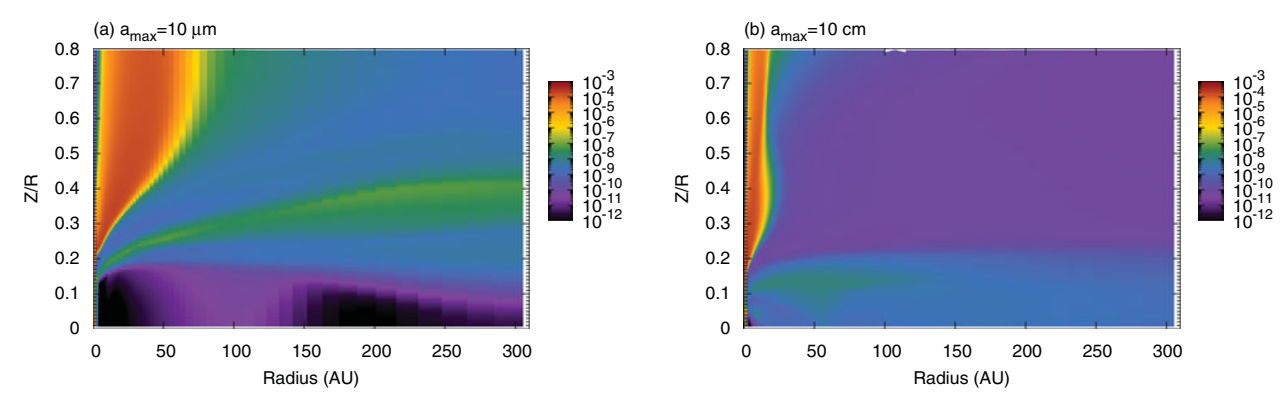

Figure 1. Water abundance profile for different dust models. $\mathrm{H}_{2} \mathrm{O}$ abundant region in the inner disk surface and $\mathrm{H}_{2} \mathrm{O}$ depleted region near the outer disk midplane shrink as grains grow.

In this case, most of the oxygen becomes water and its fractional abundance relative to total gas reaches values as high as $\sim 10^{-4}$. In the cold outer disk, these reactions are very inefficient and hydrogenation of oxygen through ion-neutral reactions produces water,

$$
\mathrm{O} \stackrel{\mathrm{H}_{3}^{+}}{\longrightarrow} \mathrm{OH}^{+} \stackrel{\mathrm{H}_{2}}{\longrightarrow} \mathrm{H}_{2} \mathrm{O}^{+} \stackrel{\mathrm{H}_{2}}{\longrightarrow} \mathrm{H}_{3} \mathrm{O}^{+} \stackrel{\mathrm{e}^{-} / \mathrm{gr}^{-}}{\longrightarrow} \mathrm{H}_{2} \mathrm{O}
$$

Since these ion-neutral reactions are not very efficient and the gas-phase water is depleted via freeze-out onto grains, its fractional abundance becomes much less than that in the inner disk, $<10^{-7}$. The water ice frozen on the grain mantle is partially photodesorbed, which is the main production source of the gas-phase water in the outer disk. Water is formed through grain-surface reactions as well, however, we find that this formation route does not affect the resulting gas-phase water abundance in the outer disk.

Taking into consideration these basic formation processes of gas-phase water, we have studied the dependence of water abundance profile on dust grain growth in a protoplanetary disk. To model the dust size distribution, we simply assume $n(a) \propto a^{-3.5}$, where $a$ is the dust radius, and adopt maximum radii of $10 \mu \mathrm{m}$ and $10 \mathrm{~cm}$. The resulting gas-phase water abundance profiles are plotted in Fig. 1, showing that as the dust grains grow, the water abundant region shrinks in the surface layer of the inner disk, while the water depleted region also shrinks near the midplane of the outer disk. This is because the grain growth affects the gas temperature and the radiation field in the disk. As the grains grow, the gas temperature drops in the inner disk surface due to the decrease of the photoelectric heating rate on grains, which results in the shrinking of the water abundant region. Meanwhile, the FUV irradiation from the central star can penetrate deeper into the disk due to the decrease in the grain opacity, which enhances the photodesorption of water ice and shrinks the water depleted region near the midplane of the outer disk.

We also study the influence of the turbulent mixing, which shows that the gas-phase water is enhanced in the inner disk surface. This is because molecular hydrogen is transported from the middle layer to the surface layer where the reactions in Eq. (3.1) produce water efficiently in the hot inner disk. The water abundance in the cold outer disk is hardly affected. In addition, we have shown that if molecular hydrogen is formed efficiently on warm grains (Cazaux \& Tielens 2004), water abundance in the hot surface layer is enhanced very efficiently due to the reactions in Eq. (3.1) (Heinzeller et al. 2011).

\section{Modelling water line emission and comparing with observations}

Making use of the obtained water abundance profiles, we have calculated water line fluxes assuming LTE for the models with different dust sizes, and compared the results with the observations. Fig. 2 shows the $63 \mu \mathrm{m}$-water-line emitting region. The line is emitted only from inner warm region, and the line emitting region shrinks as the grains 

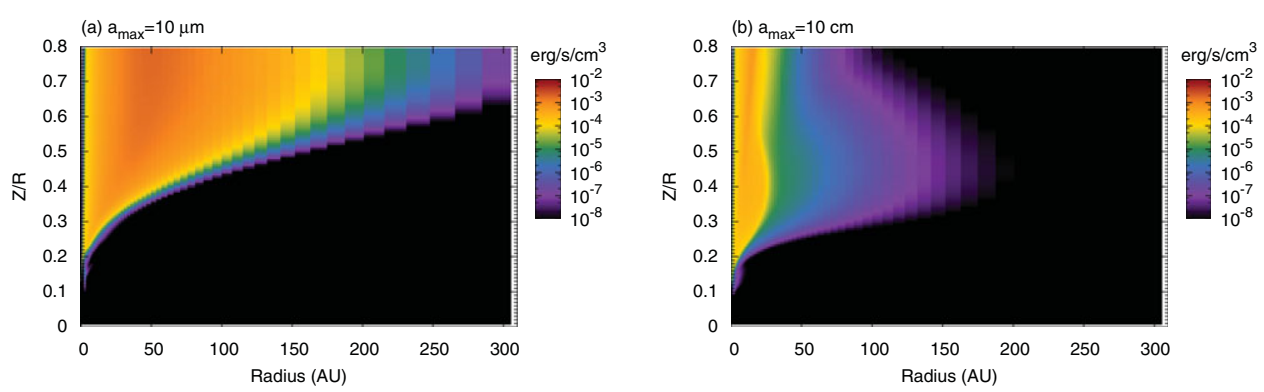

Figure 2. The $63 \mu \mathrm{m}$ water line emitting region for different dust models. The region shrinks as grains grow, which may be testable by observations of the line width.

Table 1. Calculated and observed water line fluxes at $d=140 \mathrm{pc}\left(10^{-14} \mathrm{erg} \mathrm{s}^{-1} \mathrm{~cm}^{-2}\right)$.

\begin{tabular}{|c|ccc|}
\hline Wavelength & $a_{\max }=10 \mu \mathbf{m}$ & $a_{\max }=10 \mathbf{c m}$ & observations \\
\hline $17 \mu \mathrm{m}$ (hot) & 0.19 & 0.096 & $4.8^{1}$ \\
$63 \mu \mathrm{m}$ (warm) & 0.47 & 0.23 & $0.80^{2}$ \\
$539 \mu \mathrm{m}$ (cold) & $2.6 \mathrm{e}-3$ & $6.3 \mathrm{e}-4$ & $2.7 \mathrm{e}-3^{3}$ \\
\hline
\end{tabular}

Notes: ${ }^{1}$ From Carr \& Najita (2011), AA Tau. ${ }^{2}$ From Riviere-Marichalar et al. (2012), AA Tau. ${ }^{3}$ From Hogerheijde et al. (2011), TW Hya. The distance to the disk is set at 140pc for the flux estimation.

grow because of the drop in gas temperature. The result suggests that its line width, which traces the Kepler rotation velocity at the line emitting region, could probe grain evolution in the disk surface.

Table 1 shows the calculated and observed water line fluxes at $17 \mu \mathrm{m}$ (hot), $63 \mu \mathrm{m}$ (warm), and $539 \mu \mathrm{m}$ (cold). The LTE assumption overestimates the fluxes by up to factor of 5 for the hot line (e.g., Meijerink et al. 2009). The differences between the models are not very large since the lines are optically thick. The line fluxes are slightly weaker for the model with larger grains mainly due to the drop in gas temperature. The line emitting regions shrink for the hot and warm lines, while the gas temperature of the line emitting region decreases for the cold line, which results in the weaker line fluxes. Comparing the results with the observations, the model with smaller grains is preferred, which may suggest that a certain amount of small grains can survive at least in the disk surface. The hot water line flux is a bit weaker than the observed value even for the model with small grains, but turbulent mixing can enhance the flux to match the observations. Very efficient molecular hydrogen formation on warm grains is ruled out in order to explain the observed hot/warm water lines (Heinzeller et al. 2011).

\section{References}

Carr, J. S. \& Najita, J. R. 2011, ApJ, 733, 102

Cazaux, S. \& Tielens, A. G. G. M. 2004, ApJ, 604, 222

Heinzeller, D., Nomura, H., Walsh, C., \& Millar, T. J. 2011, ApJ, 731, 115

Hogerheijde, M. R., Bergin, E. A., Brinch, C., et al. 2011, Science, 334, 338

Meijerink, R., Pontoppidan, K. M., Blake, G. A., et al. 2009, ApJ, 704, 1471

Riviere-Marichalar, P., Ménard, F., Thi, W. F., et al. 2012, A\& A, 537, L3

Walsh, C., Millar, T. J., \& Nomura, H. 2010, ApJ, 722, 1607

Walsh, C., Nomura, H., Millar, T. J., \& Aikawa, Y. 2012, ApJ, 747, 114 